\title{
ANÁLISIS DE LA DERIVADA DE PRESIÓN EN YACIMIENTOS NATURALMENTE FRACTURADOS CON FRONTERAS PARCIALMENTE ABIERTAS
}

\author{
Pressure derivative analysis for naturally-Fractured RESERVOIRS \\ WITH PARTIAL CONSTANT-PRESSURE BOUNDARY
}

Freddy Humberto Escobar

Sady Stefany Salazar"

Alfredo Ghisays-Ruiz

Recibido: 13 de diciembre de 2017

Aceptado: 23 de abril de 2018

\section{Resumen}

La complejidad de los recursos de rocas generadoras es cada vez mayor, por eso se precisa una óptima caracterización. Los modelos existentes para estudiar el comportamiento de la presión en formaciones heterogéneas consideran únicamente frontera abierta o frontera cerrada. En este trabajo se analizó la derivada de presión para un pozo vertical de petróleo en un yacimiento naturalmente fracturado con frontera externa abierta variable. La respuesta de presión está gobernada por dos parámetros: tasa final de flujo en la frontera externa $\left(q_{W I}\right)$ y tiempo en el que se inicia la invasión de fluido en la frontera externa $(\tau)$; dependiendo de la variabilidad de dichos parámetros, se presentan tres condiciones en las que actúa la frontera externa: cerrada, parcialmente abierta y abierta. Para cada caso estudiado se identificaron líneas y puntos característicos, base fundamental para generar las ecuaciones que determinan los parámetros que describen a este tipo de reservorios, como $q_{W I} \mathrm{y}$ $\tau$. Por último, se extendió la técnica TDS para interpretar pruebas de presión en estos sistemas. La metodología se verificó satisfactoriamente con ejemplos sintéticos.

Palabras claves: yacimientos naturalmente fracturados, flujo en la frontera externa, pruebas de presión.

\footnotetext{
*Ph.D. en Ingeniería de Petróleos. Profesor titular, grupo de investigación Geosciences, Infraestructure, Productiviy, Environment (GIPE), Universidad Surcolombiana, Neiva, Colombia. ORCID:https://orcid.org/0000-0003-4901-6057. fescobar@usco.edu.co

** Ing. de Petróleos, B.Sc. en Ingeniería de Petróleos. Investigadora, grupo de investigación Geosciences, Infraestructure, Productiviy, Environment (GIPE), Universidad Surcolombiana, Neiva, Colombia. stefani_0193@ hotmail.com *** Lic. en Física y Matemáticas, M.Sc. en Geofísica. Profesor, grupo de investigación Geología, Geofísica y Procesos Marino-Costeros, Universidad del Atlántico, Barranquilla, Colombia. alfghi1@gmail.com
} 


\section{Abstract}

The complexity of conventional resources is increasing, and, therefore an optimum characterization is required. Existing models to study pressure behavior in heterogeneous formations consider only either constant-pressure or closed boundaries. In this paper the pressure derivative for a vertical oil well in a naturally fractured reservoir with a variable open external boundary is analyzed. The parameters governing the pressure response are: final flow rate at the external boundary $\left(q_{W I}\right)$ and time at which begins the invasion of fluid at the external boundary $(\tau)$, depending on its variability of these parameters, there are three conditions in which the external border acts: closed, partially open and constant-pressure. Lines and characteristic points were identified for each individual case to obtain the fundamental basis to generate the equations for the determination of the parameters that describe this type of reservoirs, such as: $q_{W I}$ and $\tau$. Finally, the TDS technique was extended to interpret pressure tests in these systems. The methodology was satisfactorily verified by solving synthetic examples.

Keywords: naturallyfractured reservoirs, changing outer boundary flux, transient pressure analysis.

\section{INTRODUCCIÓN}

Hay en la literatura especializada gran cantidad de investigaciones que estudian el comportamiento de los yacimientos con flujo en la frontera externa y que están relacionados con el influjo de agua, pues la intrusión de agua proporciona una fuente importante de energía al reservorio. Van Everdinger y Hurst (1949) establecieron que la invasión de agua en la frontera externa del yacimiento actuaba como una barrera de presión constante; con este presupuesto, construyeron la solución analítica de la ecuación de difusividad en el espacio laplaciano (aplicada al espacio real por Matthews y Russell [1967]). Para el caso de un reservorio con agua de fondo, Coats (1962) diseñó un modelo de prueba de pozo en el que la frontera inferior actúa como una barrera de presión constante. Olarewaju (1989) resolvió el modelo en el que ambas fronteras (externa e inferior) actúan como una barrera de presión constante.

La idea de un modelo para describir un yacimiento naturalmente fracturado con flujo en la frontera externa parte de las premisas dadas por Warren y Root (1963), quienes estudiaron el comportamiento de los yacimientos de doble porosidad a partir de los parámetros de coeficiente de almacenaje adimensional $\omega$ y flujo interporoso $\lambda$. El flujo a través de medios porosos (considerando frontera variable) fue desarrollado por Pascal y Pascal (1985). Acosta y Ambastha (1994) propusieron una metodología para analizar una prueba de presión transitoria de yacimientos de doble porosidad.

La correcta caracterización de un yacimiento desempeña un papel importante en la toma de decisiones y alternativas para su producción. Una de las técnicas más utilizadas para evaluar un reservorio son las pruebas de presión, las cuales determinan las propiedades y predicen el desempeño del mismo.

Un artículo clásico sobre los yacimientos naturalmente fracturados es el de Warren y Root (1963). Estos autores usaron el modelo del cubo de azúcar que Engler y Tiab (1996) aplicaron para desarrollar la técnica TDS. Esta técnica es bastante versátil para caracterizar los yacimientos naturalmente fracturados, especialmente en los casos en que el primer flujo radial está enmascarado por el almacenamiento del pozo y el análisis convencional no se aplica. Desde entonces se han incorporado muchas más aplicaciones para la técnica TDS, entre las que se puede destacar: Engler 
y Tiab (1996b) extendieron la técnica de TDS a pozos horizontales; Escobar, Sánchez y Cantillo (2008) aplicaron esta técnica para el análisis de la velocidad transitoria en yacimientos homogéneos y heterogéneos; Escobar, Hernández y Saavedra (2010) investigaron detalladamente las formaciones heterogéneas; Escobar, Giraldo, Zambrano y Cantillo (2011) desarrollaron la TDS para crudo pesado en yacimientos naturalmente fracturados; Escobar, Zhao y Zhang (2014) establecieron el efecto en la frontera del gradiente de presión para pozos horizontales en yacimientos fracturados; Escobar, Camacho y Rojas (2014) estudiaron los yacimientos de doble permeabilidad-triple porosidad; Ángel, Núñez-López y Velasco-Hernández (2014) realizaron un modelo de prueba de pozo de un yacimiento con flujo variable en la frontera externa y la solución analítica de la presión en espacio real. Por su parte, Wang, Yao, Cai y Liu (2015) analizaron el comportamiento de la prueba de presión de un yacimiento naturalmente fracturado con flujo variable en la frontera externa a partir de los dos parámetros: tasa final adimensional de flujo en la frontera externa $\left(q_{R D}\right)$ y tiempo adimensional en el que se inicia la invasión de fluido en la frontera externa $\left(\tau_{D}\right)$. Estos autores tuvieron en cuenta la distribución dinámica de presión de Hsieh, Chilingar y Lin, (2007), Hsieh, Chilingar y Lin (2008) y Raghavan (1993) y el método de cálculo bajo las condiciones de frontera infinita, cerrada y a presión a constante Raghavan (1993).

Esta investigación utilizó el modelo propuesto por Wang et ál. (2015). Se formularon expresiones matemáticas exactas que determinan los parámetros que caracterizan la invasión de fluido en un yacimiento naturalmente fracturado; para ello se simularon diferentes pruebas de presión de un pozo ubicado en un yacimiento de doble porosidad con flujo variable en la frontera externa. Posteriormente, se construyeron gráficas log-log de la derivada de presión adimensional vs. tiempo para encontrar puntos característicos en el desarrollo de la técnica TDS para el problema tratado en este documento.

\section{Modelo matemático}

El modelo matemático propuesto por Wang et ál. (2015) considera las suposiciones comúnmente utilizadas en los análisis de pruebas de pozos: porosidad constante, permeabilidad constante, viscosidad constante, etc. (ver ecuación 1 ).

$$
\begin{gathered}
\bar{P}_{D}=\frac{K_{0}\left(r_{D} \sqrt{u}\right) I_{1}\left(r_{D e} \sqrt{u}\right)+I_{0}\left(r_{D} \sqrt{u}\right) K_{1}\left(r_{D e} \sqrt{u}\right)}{s \sqrt{u}\left[K_{1}(\sqrt{u}) I_{1}\left(r_{D e} \sqrt{u}\right)-I_{1}(\sqrt{u}) K_{1}\left(r_{D e} \sqrt{u}\right)\right]}+ \\
\frac{q_{D}}{r_{D e}} \frac{K_{0}\left(r_{D} \sqrt{u}\right) I_{1}(\sqrt{u})+I_{0}\left(r_{D} \sqrt{u}\right) K_{1}\left(r_{D e} \sqrt{u}\right)}{\sqrt{u}\left[K_{1}(\sqrt{u}) I_{1}\left(r_{D e} \sqrt{u}\right)-I_{1}(\sqrt{u}) K_{1}\left(r_{D e} \sqrt{u}\right)\right]}
\end{gathered}
$$

$u=s f(s)$ y $f(s)$ son definidos después de la ecuación 14 en Warren y Root (1963).

Los parámetros adimensionales utilizados para extender la técnica TDS son:

$$
t_{D}=\frac{0.0002637 k t}{\mu\left(\phi_{m} c_{m}+\phi_{f} c_{f}\right) r_{w}^{2}}
$$




$$
\begin{gathered}
t_{D A}=\frac{0.0002637 k t}{\mu \phi c_{1} A} \\
t_{D A}=\frac{t_{D} * r_{w}^{2}}{A} \\
r_{D}=r_{D e}=\frac{r_{e}}{r_{w}}
\end{gathered}
$$

La presión y derivada de presión adimensional:

$$
\begin{gathered}
P_{D}=\frac{k h}{141.2 q \mu B} \Delta P \\
t_{D}^{*} P_{D}=\frac{k h}{141.2 q \mu B} t * \Delta P
\end{gathered}
$$

Tasa final adimensional de flujo en la frontera externa:

$$
q_{R D}=\frac{q_{W I}}{q}
$$

\section{Desarrollo de la técnica tDs}

\section{Frontera cerrada o parcialmente abierta}

\section{Efecto del $q_{R D}$}

La condición de frontera externa cerrada o parcialmente abierta ocurre cuando el fluido que ingresa en la frontera externa del yacimiento es menor comparado con lo que está produciendo el pozo $\left(q_{R D}<1\right)$. La figura 1 muestra el comportamiento de la presión y derivada de presión en términos adimensionales para un yacimiento naturalmente fracturado con frontera externa cerrada $\left(0<q_{R D}\right.$ $\leq 0.3$ ). En la figura se evidencia inicialmente una cavidad en la curva de la derivada de presión que está condicionada por los parámetros $\omega$ y $\lambda$, los cuales describen la interacción de flujo entre la matriz y el sistema de fracturas. Posteriormente se forma el estado pseudoestable y parcialmente abierto $\left(0.5 \leq q_{R D}<1\right)$. Debido a la intrusión de fluido en el reservorio, se forma una doble cavidad en la curva de la derivada de presión; la primera establece el flujo entre la matriz y el sistema de fracturas, y la segunda, la invasión de fluido en el reservorio (el flujo en la frontera externa estará determinada por los parámetros $q_{R D} \mathrm{y} \tau_{D}$ ). Cuando la onda de presión llega a la frontera se presenta el estado pseudoestable. 


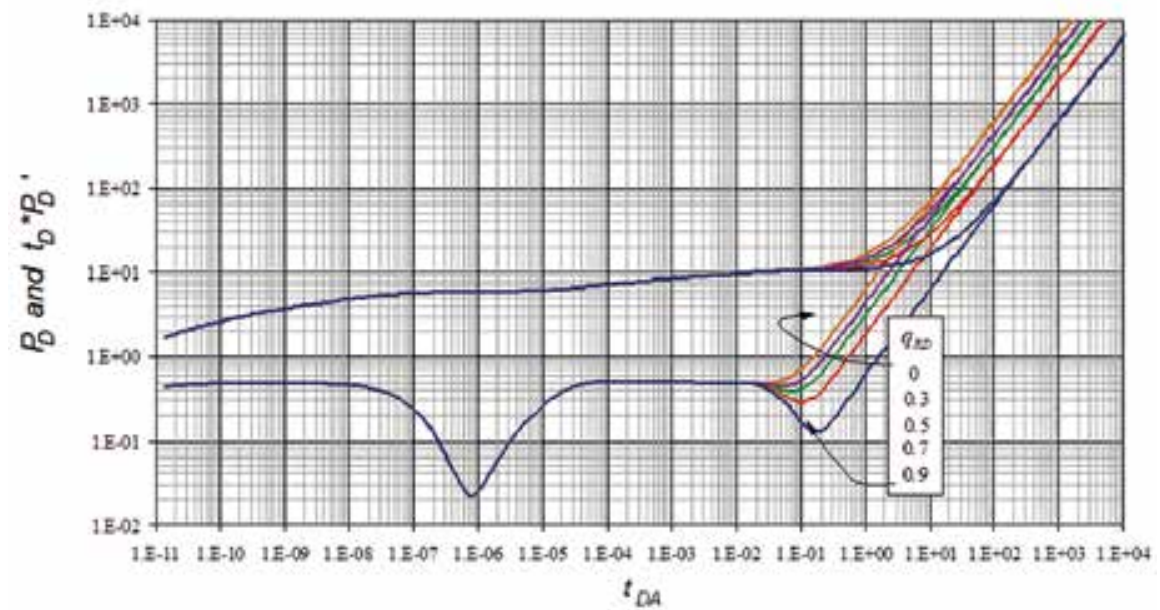

Figura 1. Presión y derivada de presión para un yacimiento naturalmente fracturado con frontera externa cerrada y parcialmente abierta.

La tasa final adimensional de flujo en la frontera externa $\left(q_{R D}\right)$ está dada por:

$$
q_{R D}=\left|1-\frac{\left(t_{D} * P_{D}^{\prime}\right)_{p s s}}{2 \pi t_{D A_{P s}}}\right|
$$

La tasa final de flujo en la frontera externa $\left(q_{W I}\right)$ es:

$$
q_{R D}=q^{*}\left|1-\frac{1}{0.233951}\left(\frac{t^{*} \Delta P^{\prime}}{t}\right)_{p s s}\left(\frac{h\left(\phi c_{t}\right)_{t} A}{q B}\right)\right|
$$

La ecuación 10 se puede utilizar tanto para la condición de frontera externa cerrada como para la parcialmente abierta, ya que están en función de un punto durante el estado pseudoestable.

Cuando la frontera externa actúa como parcialmente abierta, la intrusión de fluido en el yacimiento está representada por una cavidad; por lo tanto, a partir del punto mínimo de esta se puede calcular la tasa final de flujo que ingresa al yacimiento.

El parámetro adimensional $q_{R D}$ está dado por:

$$
\begin{aligned}
& q_{R D}=\left(1-\frac{\left(t_{D} * P_{D}^{\prime}\right)_{\min }}{0.438697}\right)^{\frac{1}{3.099}} \\
& q_{R D}=\left(1-\frac{\left(t_{D} * P_{D}^{\prime}\right)_{\min }}{0.435058}\right)^{\frac{1}{3.15}}
\end{aligned}
$$




$$
q_{R D}=\left(0.99-\frac{\left(t_{D} * P_{D}^{\prime}\right)_{\min }}{0.427986}\right)^{\frac{1}{3.5}}
$$

El parámetro $q_{W I}$ es:

$$
\begin{aligned}
& q_{W I}=q^{*}\left(1-\frac{k h\left(t^{*} \Delta P^{\prime}\right)_{\min }}{61.94402 * q \mu B}\right)^{\frac{1}{3.099}} \\
& q_{W I}=q^{*}\left(1-\frac{k h\left(t^{*} \Delta P^{\prime}\right)_{\min }}{61.43019 * q \mu B}\right)^{\frac{1}{3.15}} \\
& q_{W I}=q^{*}\left(0.99-\frac{k h\left(t^{*} \Delta P^{\prime}\right)_{\min }}{60.43162 * q \mu B}\right)^{\frac{1}{3.5}}
\end{aligned}
$$

\section{Efecto del $r_{D e}$}

El comportamiento de la derivada de presión se ve afectado por el radio adimensional del yacimiento, ya que a medida que este incrementa la onda de presión, toma más tiempo en llegar a la frontera (ver figura 2).

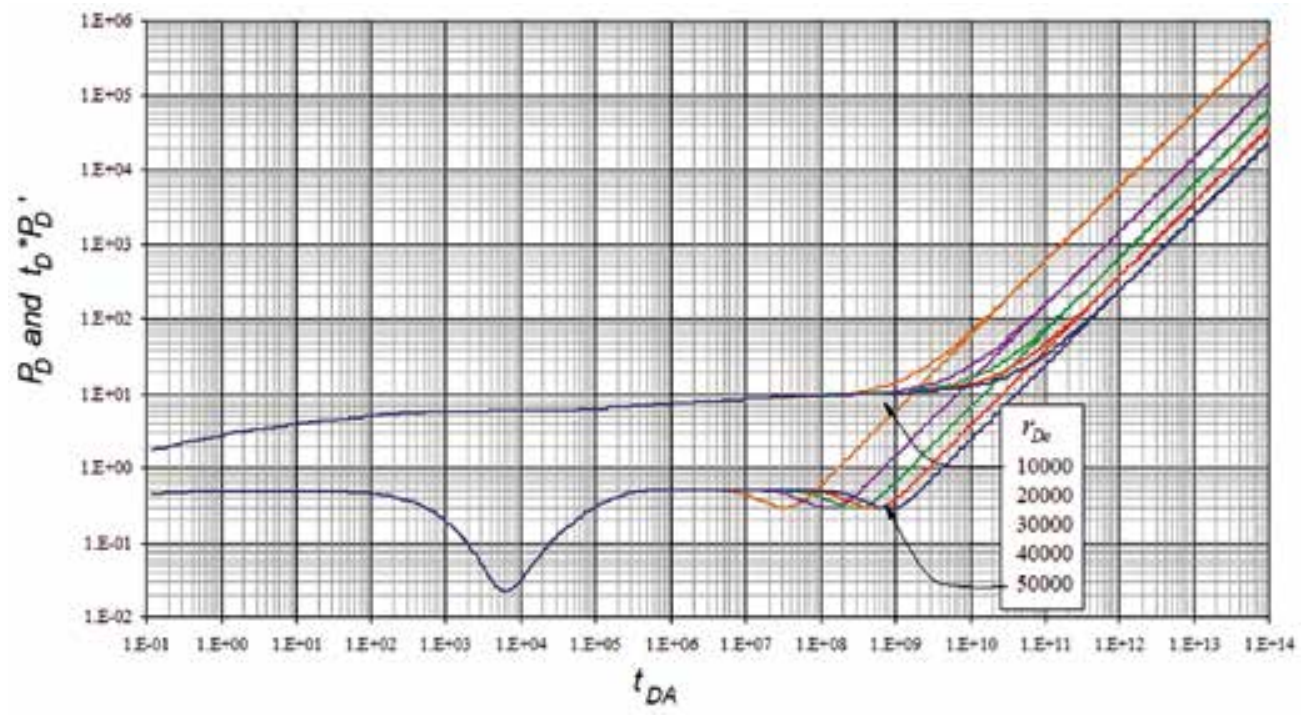

Figura 2. Efecto del $r_{\mathrm{De}}$ sobre la presión y derivada de presión en un yacimiento naturalmente fracturado con frontera externa parcialmente abierta. 
La expresión matemática que calcula el radio adimensional del yacimiento $\left(r_{D e}\right)$ a partir del $\tau_{D}$ es:

$$
r_{D e}=\left(\frac{\tau_{D}}{0.14755254}\right)^{\frac{1}{1.89}}
$$

El radio del yacimiento $\left(\mathrm{r}_{\mathrm{e}}\right)$ está dado por:

$$
r_{e}=r_{w} *\left(\frac{0.00178716 k \tau}{\phi c_{t} \mu r_{w}^{2}}\right)^{\frac{1}{1.89}}
$$

Donde $\tau$ es el tiempo de inicio de la invasión de fluido. El área se calcula con la siguiente ecuación:

$$
A=\frac{\pi r_{e}^{2}}{43.560}
$$

\section{Influencia del parámetro $\boldsymbol{T}_{D}$}

La intrusión de fluido en el yacimiento está representada por una cavidad en la curva de la derivada de presión (periodo de transición final). Su duración se ve afectada por el parámetro $\tau_{D}$, pues a medida que este incrementa, la invasión en la frontera externa se hace más lenta y la cavidad que se forma aparecerá más tarde y su amplitud será más corta (Wang et ál., 2015).

Como se observa en la figura 3 , cuando $\tau_{D}>1 \times 10^{9}$, la onda de presión alcanza más rápido la frontera del yacimiento y, como consecuencia, no aparece cavidad en la curva de la derivada de presión, sino un paso lateral que representa la invasión en la frontera externa.

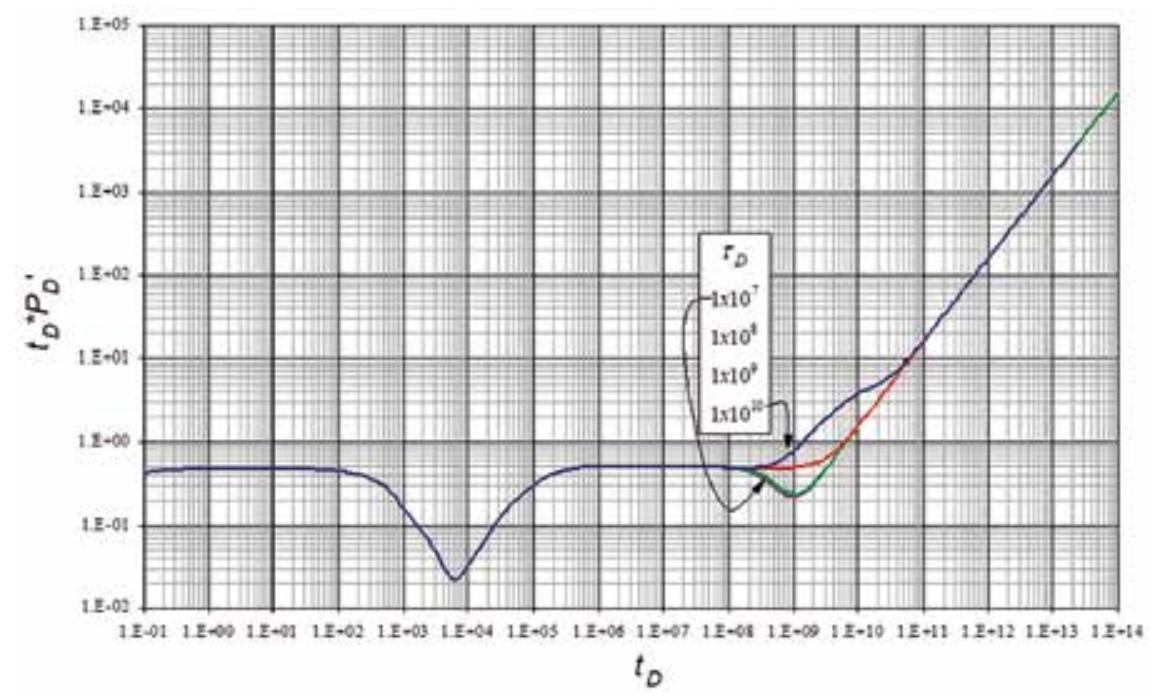

Figura 3. Influencia del $\tau_{\mathrm{D}}$ en la curva de la derivada de presión. 


\section{Frontera a presión constante}

\section{Efecto del $q_{R D}$}

Se manifiesta cuando el caudal que ingresa en la frontera externa es igual a lo que produce el pozo $\left(q_{R D}=1\right)$; por lo tanto, la presión tiende a estabilizarse. En la figura 4 se expone el comportamiento de la presión y derivada de presión en términos adimensionales para un yacimiento naturalmente fracturado con frontera externa abierta; aquí se evidencia que en la curva de presión se presenta una línea horizontal equivalente a la condición de presión constante y que en la curva de la derivada de presión se muestra el estado estable cuando la onda llega a la frontera.

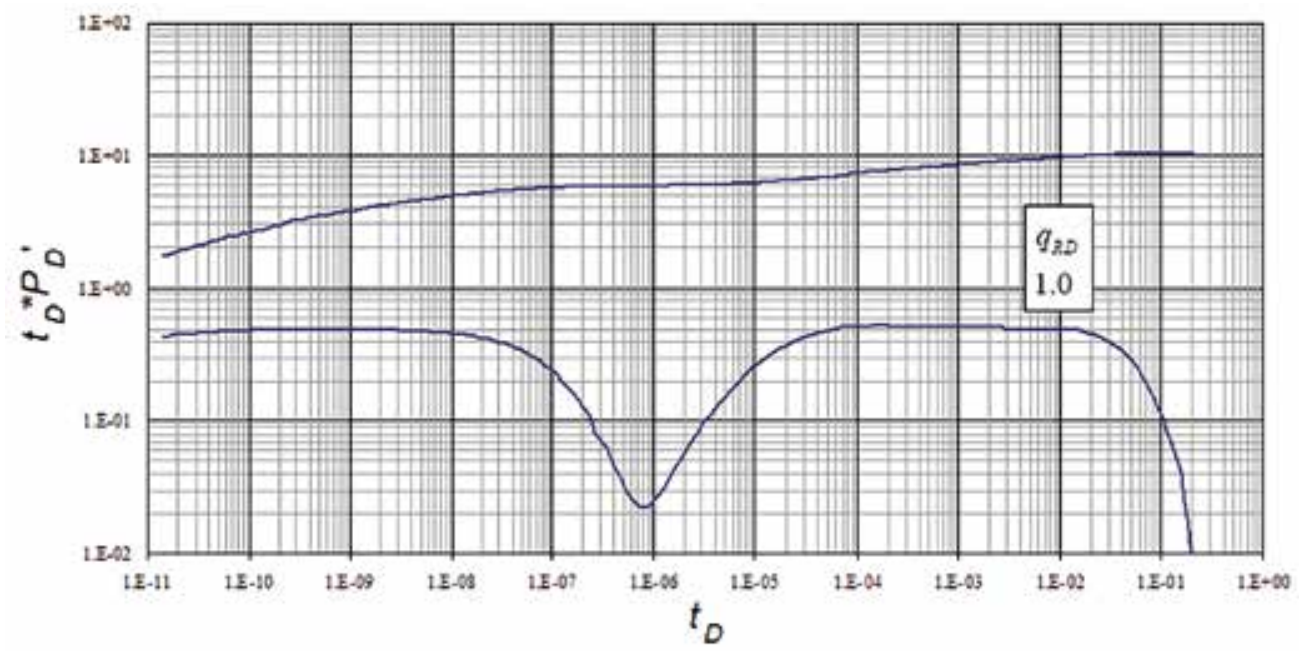

Figura 4. Presión y derivada de presión adimensional para un yacimiento naturalmente fracturado con frontera externa abierta.

Cuando se presenta el estado estable, la tasa final adimensional de flujo en el yacimiento $\left(q_{R D}\right)$ está dada por:

$$
\begin{gathered}
q_{R D}^{0.6}=\frac{0.014266}{\left(t_{D A}\right)_{s s}^{0.994} *\left(t_{D} * P_{D}{ }^{\prime}\right)_{s s}} \\
q_{R D}=\frac{0.020817}{\left(t_{D A}\right)_{s s}^{0.997} *\left(t_{D} * P_{D}^{\prime}\right)_{s s}}-0.47 \\
q_{R D}=-1.77898 * \ln \left(\frac{\left(t_{D A}\right)_{s s}^{0.995} *\left(t_{D} * P_{D}{ }^{\prime}\right)_{s s}}{0.024963}\right)
\end{gathered}
$$

La tasa final de flujo en la externa $\left(q_{W I}\right)$ es: 


$$
\begin{gathered}
q_{W I}=q^{*}\left[\frac{7270.319^{*} q \mu B}{k h\left(t^{*} \Delta P^{\prime}\right)_{s s}}\left(\frac{(\phi c)_{t} \mu A}{k t_{s s}}\right)^{0.994}\right]^{\frac{1}{0.6}} \\
q_{W I}=q^{*}\left[\frac{10874.418 * q \mu B}{k h\left(t^{*} \Delta P^{\prime}\right)_{s s}}\left(\frac{(\phi c)_{t} \mu A}{k t_{s s}}\right)^{0.997}\right]-0.47 \\
q_{W I}=q^{*}\left(-1.77898 * \ln \left(\frac{7.796 \times 10^{-5} * k h\left(t * \Delta P^{\prime}\right)_{s s}}{q \mu B}\left(\frac{k t_{s s}}{(\phi c)_{t} \mu A}\right)^{0.995}\right)\right)
\end{gathered}
$$

\section{Influencia del $r_{D e}$}

Como se esperaba, cuanto mayor sea el radio del yacimiento, la onda de presión tomará más tiempo en llegar a la frontera y el estado estable se formará más tarde (ver la figura 5). El radio del yacimiento puede estimarse con la ecuación 19.

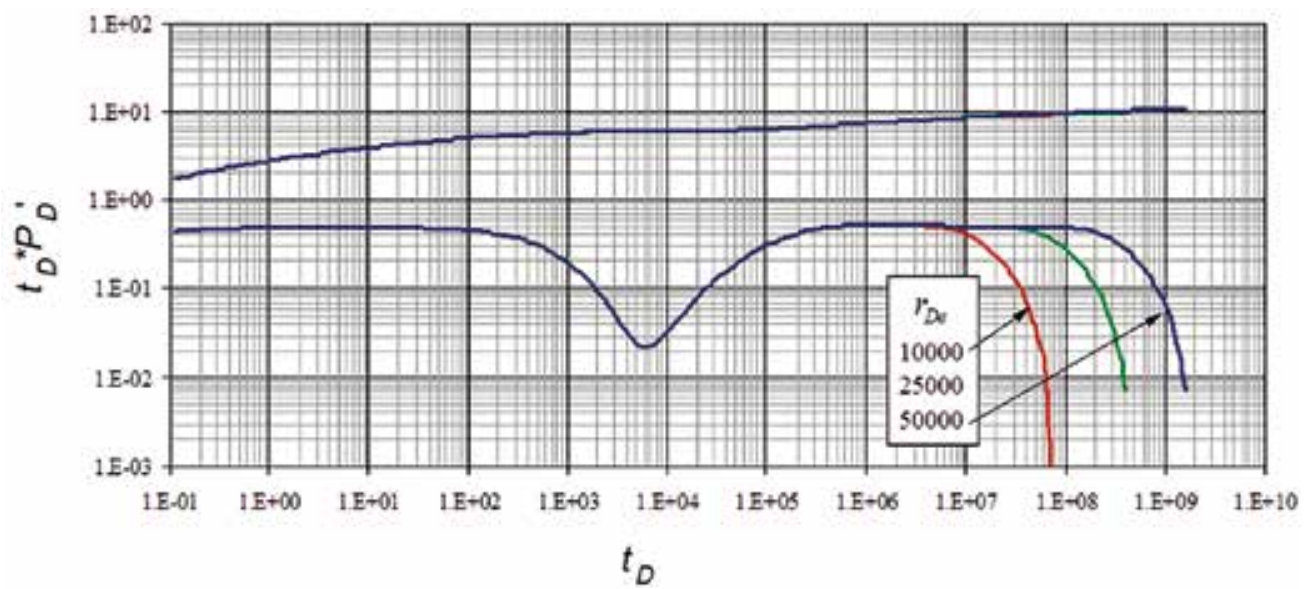

Figura 5. Efecto del $r_{D e}$ sobre la presión y derivada de presión en un yacimiento naturalmente fracturado con frontera externa abierta.

\section{Influencia del parámetro $\boldsymbol{T}_{D}$}

La intrusión de fluido en el yacimiento se ve afectada por el parámetro $\tau_{D}$. Cuando este parámetro incrementa, la invasión en la frontera externa se hace más lenta y el estado estable aparecerá más tarde. Como se observa en la figura 6 , si $\tau_{D}>1 \times 10^{9}$, la onda de presión alcanza más rápido la frontera del yacimiento y, como consecuencia, trata de formarse el estado pseudoestable en la curva de la derivada de presión, ya que no se siente el efecto del caudal que ha ingresado. Cuando el caudal que invade el yacimiento es considerable, se estabiliza la presión y, por ende, aparece el estado estable en la derivada de presión. 


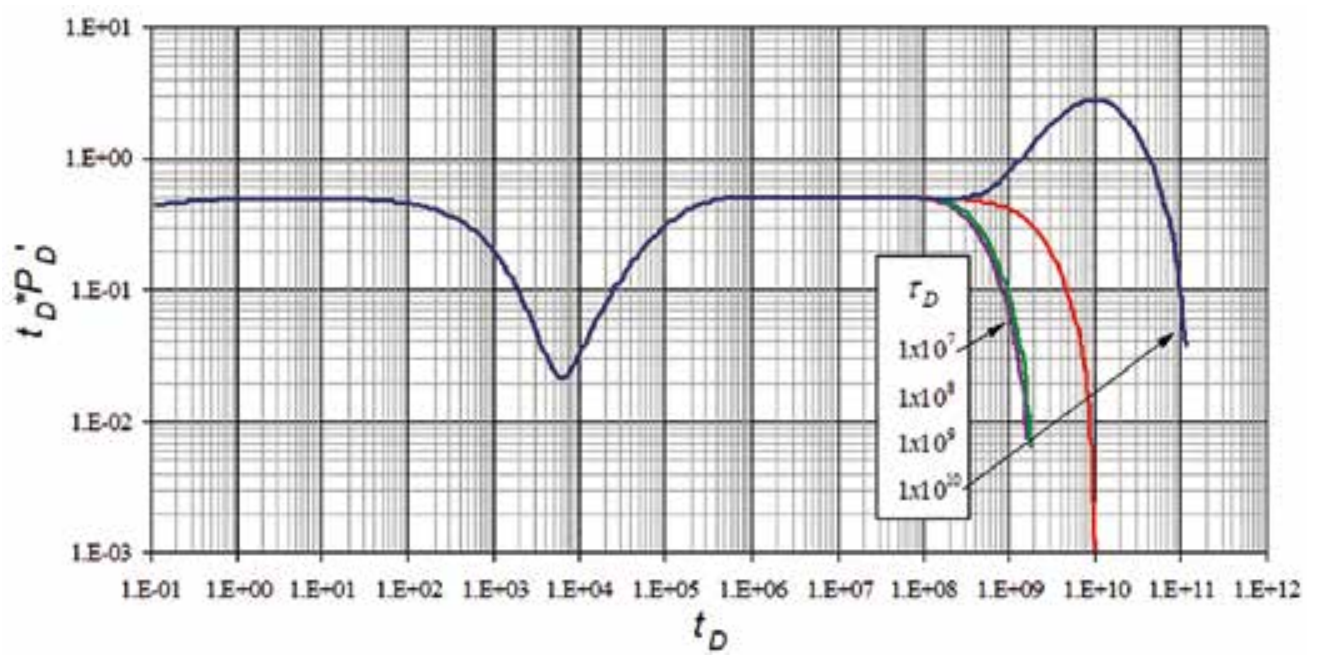

Figura 6. Influencia del $\tau_{\mathrm{D}}$ en la curva de la derivada de presión.

\section{EJEMPLOS}

La determinación de los parámetros de un yacimiento naturalmente fracturados no se presenta aquí (ver Engler y Tiab, 1996a). Es indispensable que el lector esté familiarizado con la técnica TDS; de lo contrario, se recomienda ver Tiab (1995).

\section{Ejemplo 1}

Se simuló una prueba de declinación de presión para un pozo en un yacimiento naturalmente fracturado con flujo en la frontera externa. Los datos de entrada al simulador se muestran en la tabla 1. El comportamiento de la presión y derivada de presión se presentan en la figura 7. Determinar la tasa final de flujo en la frontera externa $\left(q_{W I}\right)$ y el área del yacimiento.

Tabla 1. Datos del pozo, yacimiento y fluidos para los ejemplos 1 y 2

\begin{tabular}{lrc}
\hline \multicolumn{1}{c}{ Parámetro } & Ejemplo 1 & Ejemplo 2 \\
\hline q (bbl/D) & 560 & 800 \\
B (bbl/STB) & 1.3 & 1.25 \\
$\mu(\mathrm{cp})$ & 1.6 & 1.18 \\
$\mathrm{~h}(\mathrm{ft})$ & 29 & 75 \\
$\mathrm{rw}(\mathrm{ft})$ & 0.33 & 0.29 \\
$\phi$ & 0.12 & 0.1 \\
$\mathrm{ct}(1 / \mathrm{psi})$ & $3 \times 10-5$ & $2 \times 10-6$ \\
$\mathrm{k}(\mathrm{md})$ & 105 & 45 \\
$\mathrm{r}_{\mathrm{e}}(\mathrm{ft})$ & 9900 & 13557.5 \\
\hline
\end{tabular}




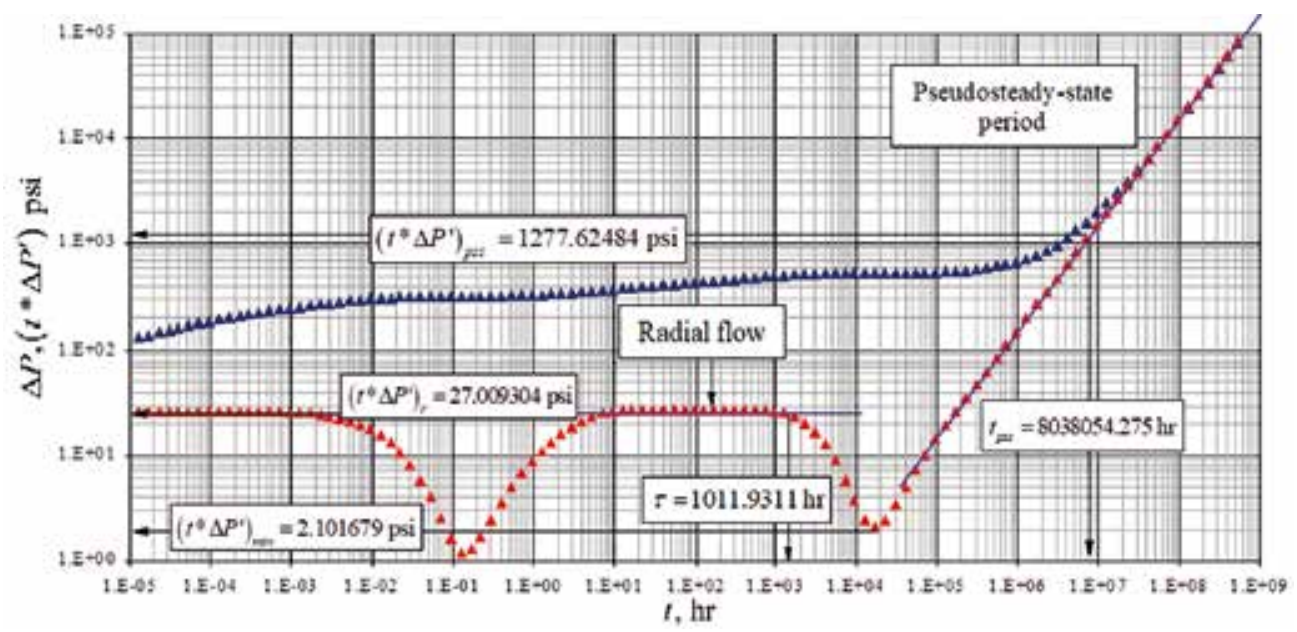

Figura 7. Log-Log de la presión y derivada de presión vs. tiempo ejemplo 1.

\section{Solución}

La siguiente información fue tomada de la figura 7:

$$
\begin{array}{lll}
t_{p s s}=8038054.275 \mathrm{hr} & \left(t^{*} \Delta P^{\prime}\right)_{p s s}=1277.62484 \mathrm{psi} & \left(t^{*} \Delta P^{\prime}\right)_{\min }=2.101679 \mathrm{psi} \\
{ }_{T}=1011.9311 \mathrm{hr} & \left(t^{*} \Delta P^{\prime}\right)_{r}=27.009304 \mathrm{psi}
\end{array}
$$

Se calcula la permeabilidad utilizando la ecuación 2.8 de Tiab (1995).

$$
k=\frac{70.6 q \mu B}{h\left(t^{*} \Delta P^{\prime}\right)_{r}}=\frac{70.6 * 560 * 1.6 * 1.3}{29 * 27.009304}=104.989 \mathrm{md}
$$

Como se forma doble cavidad en la curva de la derivada de presión, las ecuaciones que se van a utilizar son las de condición de frontera externa parcialmente abierta. Los resultados se muestran en la tabla 2.

Tabla-2. Resumen de los resultados para el ejemplo 1

\begin{tabular}{cccc}
\hline Parámetros & $\begin{array}{c}\text { Data de entrada al } \\
\text { simulador }\end{array}$ & $\begin{array}{c}\text { Ecuación } \\
\text { utilizada }\end{array}$ & Resultados \\
\hline $\mathrm{k}, \mathrm{md}$ & 105 & 2.8, Tiab (2005) & 104.99 \\
& & 10 & 543.20 \\
\multirow{2}{*}{$\mathrm{q}_{\mathrm{w},}, \mathrm{BPD}$} & 543.20 & 14 & 543.47 \\
& & 15 & 543.59 \\
& & 16 & 543.24 \\
Area, ft2 & 7068.58 & 18 & 7408.01 \\
\hline
\end{tabular}




\section{Ejemplo 2}

Se simuló una prueba de declinación de presión para un pozo en un yacimiento naturalmente fracturado con flujo en la frontera externa. Los datos de entrada al software se muestran en la tabla 1. El comportamiento de la presión y derivada de presión se presentan en la figura 8. Determinar la tasa final de flujo en la frontera externa (qWI) y el área del yacimiento.

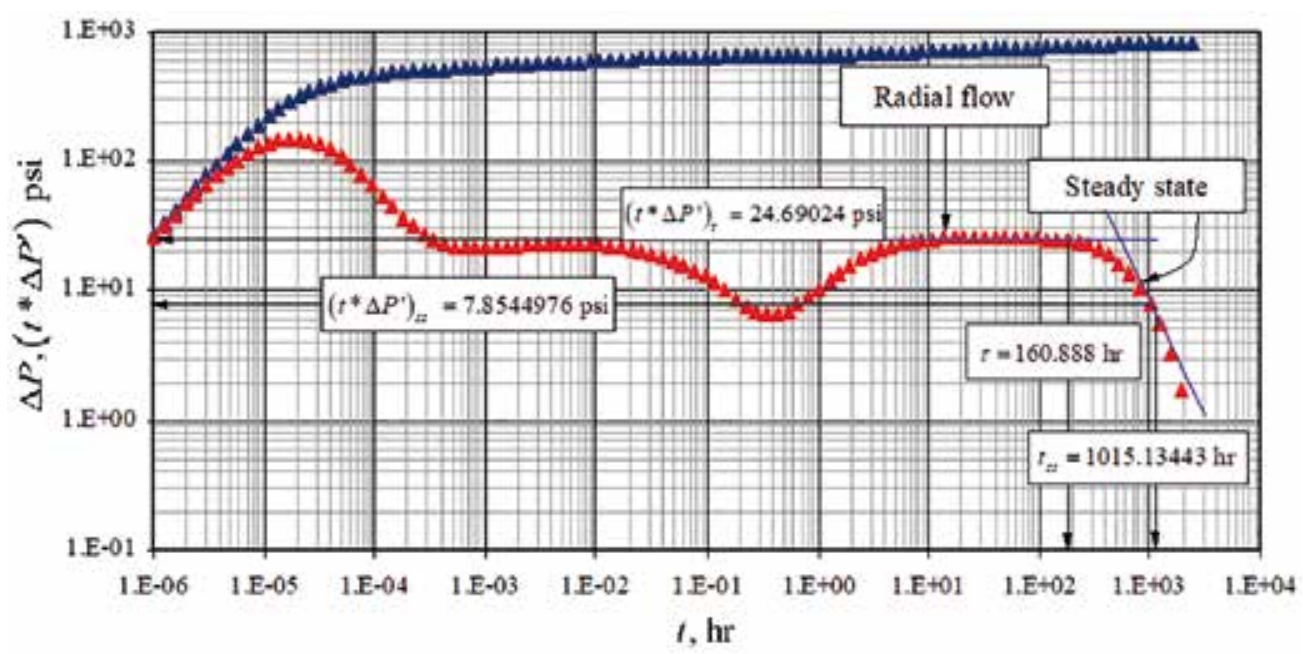

Figura 8. Log-log de la presión y derivada de presión vs. tiempo ejemplo 2.

\section{Solución}

Se traza una pendiente negativa unitaria una vez que se desarrolla el estado estable. La siguiente información fue tomada de la figura 8:

$$
\begin{aligned}
t_{s s} & =1015.13443 \mathrm{hr} \quad\left(t^{*} \Delta P^{\prime}\right)_{s s}=7.8544976 \mathrm{psi} \quad\left(t^{*} \Delta P^{\prime}\right)_{r}=24.690242 \mathrm{psi} \\
{ }_{T} & =160.888 \mathrm{hr}
\end{aligned}
$$

Como se observa en la figura 8, la presión tiende a estabilizarse y aparece el estado estable en la curva de la derivada de presión; por lo tanto, las ecuaciones que se utilizan son las de condición de frontera abierta. Los resultados se muestran en la tabla 3.

Tabla-3. Resumen de resultados para el ejemplo 2

\begin{tabular}{cccc}
\hline Parámetros & $\begin{array}{c}\text { Data de entrada al } \\
\text { simulador }\end{array}$ & $\begin{array}{c}\text { Ecuación } \\
\text { utilizada }\end{array}$ & Resultados \\
\hline $\mathrm{k}(\mathrm{md})$ & 45 & 2.8 Tiab (2005) & 44.99 \\
& & 23 & 800.32 \\
$q_{w l}(B P D)$ & 800 & 24 & 800.17 \\
& & 25 & 800.09 \\
Area (ft2) & 13256.27 & 24 & 12880.30 \\
\hline
\end{tabular}




\section{ANÁLISIS DE LOS RESULTAdOS}

Los resultados obtenidos en los ejemplos son bastante cercanos a los de la simulación. Es importante resaltar que para determinar la tasa final de flujo en la frontera externa, la mayor exactitud la arroja la ecuación 10. Para calcular el área del yacimiento a partir del tiempo en que se inicia la invasión en la frontera externa es indispensable seleccionar correctamente este punto, ya que la ecuación es muy sensible a cualquier cambio en dicho valor.

\section{Conclusiones}

La intrusión de fluido en los yacimientos naturalmente fracturados genera un comportamiento característico en la curva de la derivada de presión, en la que se identifica la condición en la que actúa la frontera externa: cerrada, parcialmente abierta y abierta; condiciones que fueron modeladas en esta de investigación.

Se desarrollaron ecuaciones que determinan los parámetros que caracterizan la invasión de fluido en la frontera externa de un yacimiento de medio dual. En el análisis de los efectos que produce la variabilidad de los parámetros involucrados en dichas expresiones, se observó que en los rangos de $q_{R D}$ de 0-0.98 en la curva de la derivada de presión se presenta el estado pseudoestable, el cual se ajusta a una pendiente unitaria. Por su parte, cuando $q_{R D}=1$, se forma el estado estable, que se identifica por una pendiente negativa unitaria.

\section{SímBolos Y ABREVIATURAS}

\section{Nomenclatura}

$\begin{array}{ll}B & \text { Factor de volumétrico del aceite, bbl/STB } \\ k & \text { Permeabilidad, } \mathrm{md} \\ c_{t} & \text { Compresibilidad total, } 1 / \mathrm{psi} \\ b & \text { Espesor de la formación, } \mathrm{ft} \\ A & \text { Área de drenaje, } \mathrm{ft}^{2} \\ I 0 & \text { Función de Bessel } I \text { de orden } 0 \\ I 1 & \text { Función de Bessel } I \text { de orden } 1 \\ K 0 & \text { Función de Bessel } K \text { de orden } 0 \\ K 1 & \text { Función de Bessel } K \text { de orden } 1 \\ q & \text { Tasa de flujo, BPD } \\ q_{W I} & \text { Tasa final de flujo en la frontera externa, BPD } \\ r_{w} & \text { Radio del pozo, ft } \\ r_{e} & \text { Radio de drenaje, } \mathrm{ft} \\ r_{D} & \text { Radio adimensional } \\ r_{D e} & \text { Radio adimensional externo } \\ s & \text { Daño } \\ q_{R D} & \text { Tasa final adimensional de flujo en la frontera externa } \\ T & \text { Tiempo en el cual se inicia la invasión de fluido en la frontera externa }\end{array}$


$T_{D} \quad$ Tiempo adimensional en el cual se inicia la invasión de fluido en la frontera externa

$P_{D} \quad$ Presión adimensional

$t \quad$ Tiempo, hr

$t_{D} \quad$ Tiempo adimensional basado en radio del pozo

$t_{D A} \quad$ Tiempo adimensional basado en el área de drenaje

$t_{D}{ }^{*} P_{D}{ }^{\prime} \quad$ Derivada de presión adimensional

$t^{*} \Delta P^{\prime} \quad$ Derivada de presión, psi

u Operador de Laplace

$\Delta P \quad$ Delta de Presión, psi

\section{Sufijos}

$\begin{array}{ll}r & \text { Radial } \\ D & \text { Adimensional } \\ w & \text { Pozo } \\ s s & \text { Estado estable } \\ \text { pss } & \text { Estado pseudoestable } \\ \text { min } & \text { Punto mínimo }\end{array}$

\section{Símbolos griegos}

$w \quad$ Coeficiente adimensional de almacenaje, $\left(\phi c_{t}\right) f f\left[\left(\phi c_{t}\right)_{\mathrm{m}}+\left(\phi c_{t}\right)_{f}\right]$

$\Delta \quad$ Delta

$\phi \quad$ Porosidad, fracción

$\mu \quad$ Viscosidad, cp

$\lambda \quad$ Parámetro de flujo interporoso

\section{REFERENCIAS}

Acosta, L., y Ambastha, A. (1994, 1 de enero). Thermal Well Test Analysis Using an Analytical Multi-Region Composite Reservoir Model. En Society of Petroleum Engineers, SPE Annual Technical Conference and Exhibition. Conferencia llevada a cabo en Neva Orleans, Luciana, EE. UU. doi: 10.2118/28422-MS

Barenblatt, G., Zheltov, Y., y Kochina, I. (1960). Basic concepts in the theory of seepage of homogeneous liquids in fissured rocks (strata). Journal of applied mathematics and mechanics, 24(5), 1286-1303.

Del Angel, Y., Núñez-López, M., y Velasco-Hernández,J. (2014). Pressure transient analysis with exponential and power law boundary flux. Journal of Petroleum Science and Engineering, 121, 149-158.

Engler, T., y Tiab, D. (1996a). Analysis of pressure and pressure derivative without type curve matching, 4. Naturally fractured reservoirs. Journal of Petroleum Science and Engineering, 15(2-4), 127-138. 
Engler, T., y Tiab, D. (1996b). Analysis of pressure and pressure derivative without type-curve matching, 5. Horizontal well tests in naturally fractured reservoirs. Journal of Petroleum Science and Engineering, 15(2-4), 139-151.

Escobar, F., Sanchez, J., y Cantillo, J. (2008). Rate transient analysis for homogeneous and heterogeneous gas reservoirs using the TDS technique. Ciencia, Tecnologia y Futuro, 4(4), 45-59.

Escobar, F., Hernandez, D., y Saavedra, J. (2010). Pressure and pressure derivative analysis for long naturally fractured reservoirs using the TDS technique. Dyna, 77(163), 102-114.

Escobar, F., Zambrano, A., Giraldo, D., y Cantillo, J. (2011). Pressure and pressure derivative analysis for non-newtonian pseudoplastic fluids in double-porosity formations. Ciencia, Tecnologia y Futuro, 5(3), 47-59.

Escobar, F., Zhao, Y., y Zhang L. (2014). Interpretation of pressure tests in horizontal wells in homogeneous and heterogeneous reservoirs with threshold pressure gradient. Journal of Engineering and Applied Sciences, 9(11), 2220-2228.

Escobar, F., Camacho, R., y Rojas, J. (2014). Pressure and pressure derivative analysis for tripleporosity and dual-permeability systems in naturally fractured vuggy reservoirs. Journal of Engineering and Applied Sciences, 9(12), 2500-2512.

Hsieh, B., Chilingar, G., y Lin, Z. (2007). Propagation of radius of investigation from producing well. Energy Sources, Part A, 29(5), 403-417.

Hsieh, B., Chilingar, G., y Lin, Z. (2008). Determination of the constant coefficient in pressure propagation equation. Energy Sources, Part A, 30(13), 1223-1232.

Mathews, C., y Russell, D. (1967). Pressure buildup and flow tests in wells (SPE Monograph Series, vol. 1). Dallas, EE. UU.: Society of Petroleum Engineers of AIME

Pascal, H., y Pascal, F. (1985). Flow of non-Newtonian fluid through porous media. International Journal of Engineering Science, 23(5), 571-585.

Tiab, D. (1993, 1 enero). Analysis of Pressure and Pressure Derivatives Without Type-Curve Matching: I-Skin and Wellbore Storage. En Society of Petroleum Engineers, SPE Production Operations Symposium. Conferencia llevada a cabo en Oklahoma, EE. UU. doi: 10.2118/25426-MS.

Tiab, D. (1995). Analysis of pressure and pressure derivative without type-curve matching: I-skin and wellbore storage. Journal of Petroleum Science and Engineering, 12(3), 171-181.

Van Everdingen, A., y Hurst, W. (1949). The application of the laplace transformation to flow problems in reservoirs. Journal of Petroleum Technology, 1(12), 305-324b. doi:10.2118/949305-G.

Wang, D., Yao, J., Cai, M., y Liu, P. (2015). Transient pressure and productivity analysis in dual medium reservoir with changing outer boundary flux (artículo inédito remitido para su publicación a Journal of Natural Gas Science Engineering).

Warren, J., y Root, P. (1963). The Behavior of Naturally Fractured Reservoirs. Society of Petroleum Engineers Journal, 3(3), 245-255. doi: 10.2118/426-PA. 
